\title{
The Reciprocating Rectilinear Moving Mechanism at High Frequency with Low Load Driven by Two Symmetrical Gears
}

\author{
Ke-Ming SHI ${ }^{1}$, Ming-Di WANG ${ }^{1 *}$, Kang-Min $\mathrm{ZHONG}^{1}$ and Jie $\mathrm{CHEN}^{2}$
}

1School of Mechanical and Electrical Engineering, Soochow University, Suzhou, 215104, China 2Department of Mechano-electranic Engineering, Suzhou Vocational University, Suzhou, 215104, China

*Correspondence: wangmingdi@suda.edu.cn

Keywords: High frequency, Low load, Symmetrical gears, Reciprocating rectilinear moving.

\begin{abstract}
In many occasions of the practical engineering, the equipment of reciprocating rectilinear movement at high frequency is used commonly, which usually is completed by the slider-crank mechanism. But there is big friction force between the guide rail and the block in this mechanism, thus the big friction loss can be caused. When working in a long time, the mechanism will be heated and distorted and a big torque will be caused in the guide rail. Subsequently, the vibration will be generated when the slider-crank mechanism moves at high frequency. In view of these limitations, the reciprocating rectilinear moving mechanism at high frequency with low load driven by two symmetrical gears is innovated. The innovated mechanism is quite symmetrical, hence the friction force between the guide rail and the block can be almost removed and the vibration can be eliminated to a great extent as well. Additionally, the mechanical properties and motion characteristics of the new mechanism are analyzed. Compared with the slider-crank mechanism, this new mechanism is proved to have a simpler structure and better properties.
\end{abstract}

\section{Introduction}

With the rapid development of economy, the communications industry gets swift and violent progress. The competitiveness of products is determined by a series of factors, such as appearance, lifespan and price and so on. Take the laptop as an example, each of its data wires, consisting of twisted thin wires and a insulating sheath, gets bent back and forth frequently. If things go on like this, such a movement will lead to a sudden break of data wires. And the stable signal transmission will be influenced. In order to ensure the reliability and stability of this kind of wires, it must be tested by high frequency fatigue machine before it is ready for use. The common way to measure the fatigue property of data wires is to make it bent by 90 degrees and count times of bending. However, this kind of testing machine and method cannot inflect the sound fatigue property and reliability under the circumstance of high frequency and less amplitude.

In the early years, the fatigue machines manufactured by Switzerland, England, German, and Japan et al. were in the main type of electromagnetic excitation resonance [1]. With the development of electro-hydraulic servo technology, all kinds of fatigue machines were produced for the sake of various application. In 1957, a fatigue machine consisting of electro-hydraulic servo harmonic vibration system was innovated, which had many superior properties, such as high load, high frequency and low power consumption. This machine could not only cut down test time, but also save testing charge. In 1960s, the research of high frequency fatigue machine was gained increasing attention gradually. For example, the first high frequency fatigue machine was manufactured by Changchun material testing machine Research Institute successfully in 1965. The whole machine was made of autonomous system which could start and come to a steady state autonomously. The nonlinear elements in the system were elastic vibration system and the resonant frequency of autonomous vibration system was the same as elastic vibration system's. In the 1980s, the further development of high frequency machine was promoted by the rapid development of machinery. An ultra high frequency fatigue machine was manufactured by Tokyo koki seizosho, ltd. The maximum stress was $65 \mathrm{~kg} / \mathrm{cm} 2$ and the frequency ranged from 15000 to $30000 \mathrm{~Hz}$. The fatigue machine consisting of piezoelectric quartz oscillator was developed in France, which 
possessed a frequency of $92000 \mathrm{~Hz}$. The type of 20HFP1430 high frequency fatigue machine was produced by Germany AMSLER. The autonomous system of this machine was installed inside the base and the main frame was lower, by this way the stability of machine was promoted. The frequency ranged from 50 to $250 \mathrm{~Hz}$ and the maximum amplitude was 士 $1.4 \mathrm{~mm}$. The type of TESTRONIC7001 and MIKROTRON654 high frequency fatigue machine with resonance system was manufactured by Switzerland Rum. The frequency of the two machines ranged from 40 to 300 $\mathrm{Hz}$ and 40 to $230 \mathrm{~Hz}$, respectively. The type of 1603 electromagnetic resonance fatigue machine with a novel structure was innovated by INSTRON CORPORATION. The novel structure consisted of two samples that could generate inverse vibration and the massive base was removed in comparison with former's. Besides the peek load, average load and frequency could be displayed by digital.

At present, the share of hydraulic servo, electromagnetic and mechanical fatigue machine are much more than others in the market. Specifically, each of the three machines has its own advantages and disadvantages. The hydraulic servo machine is a kind of pretty advanced testing equipment. However, it costs too much and the power consumption is big when running at low frequencies. The electromagnetic machine is mainly used to test given materials that have a better fatigue property due to the high working frequency. The mechanical machine with low cost and power is appropriate for the test which has no high demand for frequency. However, the traditional mechanical fatigue machines also have many drawbacks, such as the rather complicated judging criteria of the end of the test, the slow response of the end of the test and the narrow range of output load. Thus the selection of a suitable fatigue machine is quiet important, because it can not only improve testing efficiency, but also shorten testing period.

The slider-crank mechanism is the basic structure of mechanical fatigue machine, as shown in Figure 1. The force $\mathrm{FAB}$ produced by connecting rod $\mathrm{AB}$ acts on the slider. The horizontal component of FAB brings the slider to move and the vertical component of FAB generates friction on the guide rail, which will cause friction loss and large torque on the guide rail. Therefore, violent shake may be produced due to the high frequency.

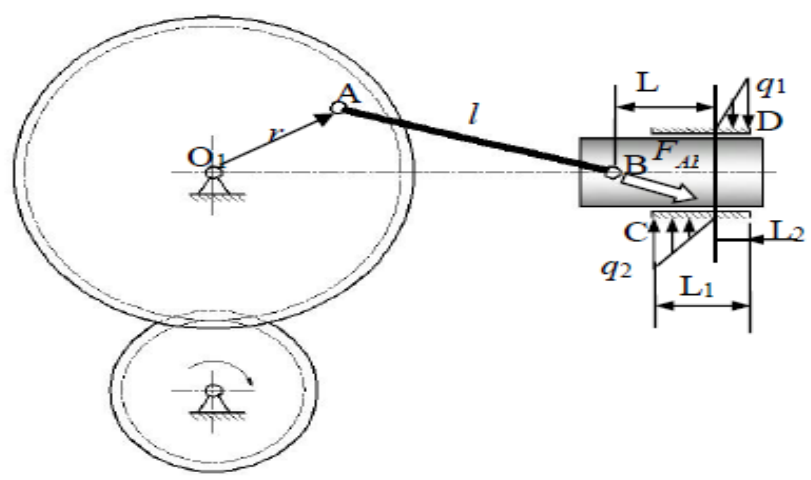

Figure 1 . The fatigue machine driven by slider-crank mechanism

In view of these limitations, the reciprocating rectilinear moving mechanism at high frequency with low load driven by two symmetrical gears comes forward in this paper. Given the symmetrical structure of the machine, there will be no friction between the connecting rod and guide rail in the theory. Under the circumstances, not only can the friction loss be reduced, but also reciprocating rectilinear movement be realized by such a mechanism.

\section{Operating Principle}

The operating principle of the reciprocating rectilinear moving mechanism driven by two symmetrical gears is shown in Figure 2. This mechanism is very simple and just contains a big gear, a pair of symmetrical gears and an output rod. The big gear is driven by electric motor directly. The small gear 1 gets revolved by engagement with the big one. In comparison with the small gear 1 , 
the small gear 2 rotates in the opposite direction completely because of the exactly symmetrical installment. The output rod moves back and forth at high frequency so as to make it possible to test the data wires owing to the rolling bearings, which is installed in small gears symmetrically.

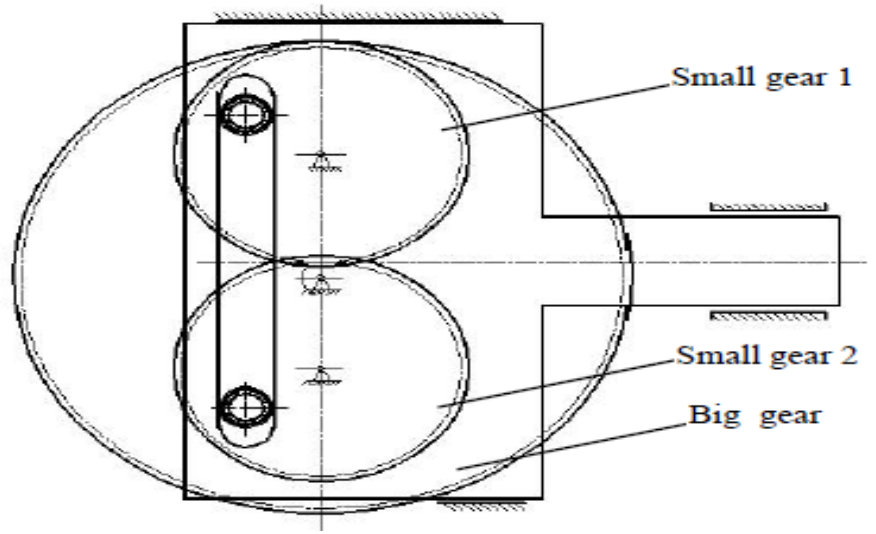

Figure 2. The fatigue machine driven by symmetrical gears

\section{Mechanical Analysis}

As shown in Figure 1, The uniformly distributed load on the slider will be produced by the guide rail of slider-crank mechanism. According to the document [2], the friction force between slider and guide rail can be deduced as:

$$
F_{f}=\frac{F_{A B y}}{2} \cdot \frac{36 L^{2}+L_{1}^{2}}{12 L L_{1}} \cdot f
$$

where FABy is vertical component of the force that is generated by the interaction between connecting rod and slider. The $f$ represents coefficient of friction between connecting rod and slider.

Additionally, the inverse force caused by uniformly distributed load $\mathrm{q} 1$ and $\mathrm{q} 2$ will produce a torque on the guide rail, which can be obtained as:

$$
M=F_{A B y}\left(L+\frac{L_{1}}{2}\right)
$$

If there is a wider gap between the guide rail and the slider, the concentrated stress will be caused between the reference point $\mathrm{C}$ and $\mathrm{D}$, as shown in Figure 1. Then the torque can be generated by the concentrated stress. The deduction of this formula is relatively easy, no complicated introduction will be stated here.

The reduction gear will be added between electrical motor and slider-crank mechanism due to the loss of friction and vibration. The frequency can be reduced and the test time can be extended by this way.

The reciprocating rectilinear moving mechanism driven by two symmetrical gears is completely symmetrical, there will be no stress and torque between rod and guide rail in theory. That is to say there will be no friction loss and vibration as well. In this case, the frequency of reciprocating move and test time can be improved to a great extent.

\section{Motion Analysis}

The radius of small gear and large gear are $\mathrm{r} 2$ and $\mathrm{R} 2$ respectively. The distance between the center of the small gear and the center of rolling bearing is represented as e. The center of the gear is taken as the reference point, and the kinematic equation of organization shown in Figure 2 can be expressed as:

$$
x=-e \sin \frac{R_{2}}{r_{2}} w t
$$

After second derivative, acceleration equation can be gained as: 


$$
a=e \frac{R_{2}^{2}}{r_{2}^{2}} w^{2} \sin \frac{R_{2}}{r_{2}} w t
$$

The w above represents output angular velocities of electrical motor.

The kinematic equation and acceleration equation of slider-crank mechanism are listed as follows[3]:

$$
\begin{aligned}
& x \approx l\left[1-\left(\frac{r}{2 l}\right)^{2}\right]+r\left[\cos \frac{r_{1}}{R_{1}} w t+\frac{r}{4 l} \cos \left(2 \frac{r_{1}}{R_{1}} w t\right)\right] \\
& a=-r \frac{r_{1}^{2}}{R_{1}{ }^{2}} w^{2}\left[\cos \frac{r_{1}}{R_{1}} w t+\frac{r}{l} \cos \left(2 \frac{r_{1}}{R_{1}} w t\right)\right]
\end{aligned}
$$

By comparing the two groups formula, we can see that the maximum travel of the reciprocating rectilinear moving mechanism driven by two symmetrical gears is $2 \mathrm{e}$. Compared with the maximum travel $2 \mathrm{r}$ of the slider-crank mechanism, there is not much difference. The maximum acceleration can be gained in the limit position, the acceleration of slider-crank mechanism is bigger obviously.

\section{Calculation}

As we can know from the analysis above, the slider-crank mechanism can only be operated at a lower speed because of the existence of lateral pressure on the guide rail. When the mechanism works at a high speed, there will be violent vibration. However, the reciprocating rectilinear moving mechanism driven by two symmetrical gears can be operated at a very high speed because there is almost no lateral pressure between the output rod and guide rail. That is to say the new mechanism can be designed as a speed-growth device.

For the purpose of getting a flagrant comparison of properties of the two organizations, specific calculation will be implemented. The parameters for motor speed, gear transmission ratio and fatigue test time are 1500r/min, 1:3 and 107, respectively. The slider-crank mechanism is speed decreasing mechanism as shown in Figure 1. The time of one bending is $0.12 \mathrm{~s}$ and the time of an entire fatigue test is 13.89 days by calculation.

On the contrary, the reciprocating rectilinear moving mechanism driven by two symmetrical gears is a speed-growth device. The time of one bending is $0.01 \mathrm{~s}$ and the time of an entire fatigue test is 1.54 days by calculation.

Thus it can be seen apparently that the reciprocating rectilinear moving mechanism driven by two symmetrical gears can improve the efficiency to a great extent by comparison with the slidercrank mechanism.

\section{Conclusions}

By analyzing the performance of mechanism and motion, it is apparent that the new mechanism has a lot of advantages, such as simple structure, uncomplicated principle, less friction, high running frequency and high efficiency.

Compared with some present fatigue machines [4-5], this new machine costs less and is more suitable for various practical engineering applications.

The reduction of friction is very effective to improve the organization performance while the load is far less than the friction.

We applied the new organization into the design and manufacturing of high frequency fatigue machine, which proves the new organization to be superior than the traditional one.

In addition, the new organization can also be applied in other occasions, such as the situation that needs the conversion of rotation and reciprocating moving.

\section{Acknowledgments}

Supported by National Natural Science Foundation of China (Grant No. 51675360) and Jiangsu Provincial Natural Science Foundation of China BK20151194. 


\section{References}

1. Qinghua $\mathrm{Xu}$, et al. A rotating mechanism for reciprocating motion. Coal Mine Machinery (in china), 28(2): 133-134 (2007)

2. Qiang Song, et al. Correction of mechanical model for sliding column in toggle force amplifier. Journal of Shandong College of Construction Engineering (in china), 15(3): 69-71 (2000)

3. Zhihua Feng, et al. Engineering Mechanics. Soochow University Press (2003)

4. Ping Zeng, Jianming Wen, et al. Research on novel inertial piezoelectric actuator. Optics and precision engineering (in china), 14(4): 623-627 (2006)

5. Taijiang Peng, et al. New Type of Accurate-motion Mechanism with Hydro-piezoelectric Drive. Chinese Journal of agricultural machinery (in china), 36(2): 51-53,60 (2005) 